\title{
Acoustic analysis of swallowing sounds in tracheostomized patients affected by traumatic brain injury
}

\author{
Raquel Guidotti Lemos ${ }^{1}$ \\ https://orcid.org/0000-0002-5324-0716 \\ Talita Todeschini Vieira ${ }^{1}$ \\ https://orcid.org/0000-0002-6638-9114 \\ Karina de Fátima Portela de Oliveira Pereira ${ }^{1}$ \\ https://orcid.org/0000-0001-5626-8020 \\ Adriano de Souza Pereira ${ }^{1}$ \\ https://orcid.org/0000-0002-5685-8300 \\ Bianca Simone Zeigelboim ${ }^{1}$ \\ https://orcid.org/0000-0003-4871-2683
}

Rosane Sampaio Santos ${ }^{1}$ https://orcid.org/0000-0001-6400-5706

Universidade Tuiuti do Paraná, Curitiba, Paraná, Brasil.

Conflict of interests: Nonexistent

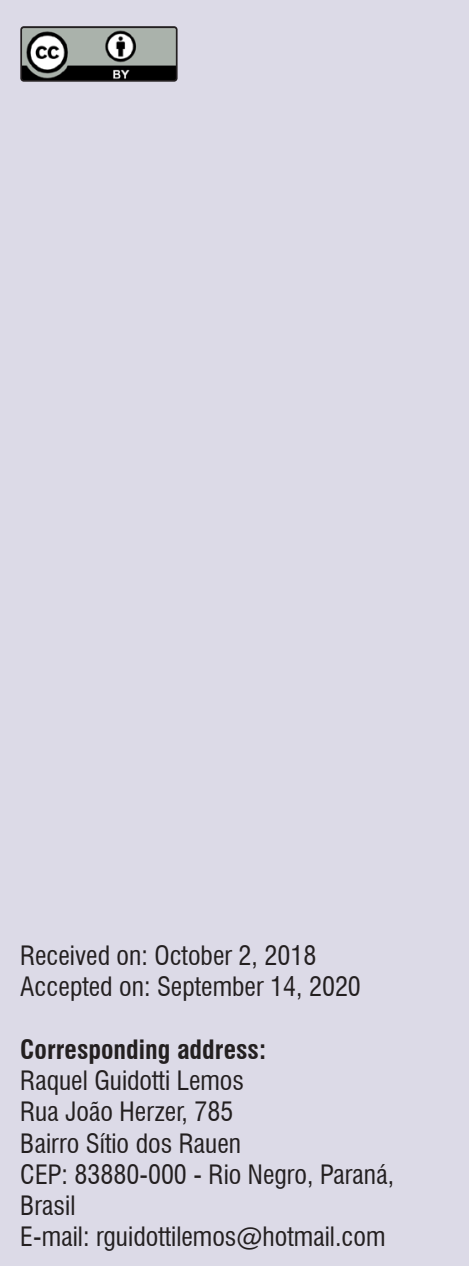

\section{ABSTRACT}

Purpose: to demonstrate the feasibility of using the acoustic analysis of swallowing sounds as a combined method in the clinical assessment of tracheostomized patients affected by traumatic brain injury.

Methods: an observational, cross-sectional study. A total of 10 adult patients, in the mean age of 43.6 years, participated. They were hospitalized in wards, semi-intensive, or intensive care units, from May to July 2016. The inclusion criterion involved being affected by traumatic brain injury, confirmed with a CT scan. The Speech Therapy Tracheal Decannulation Protocol was used in the clinical assessment, as well as the cervical auscultation with the Doppler sonar. Fisher's test was used, resulting in no significant relationship $(p>0.05)$ between the variables analyzed in the two consistencies and decannulation.

Results: the presence of an acoustic signal of laryngeal elevation was observed, as well as noise between the swallowings, and acoustic signal suggestive of residue in $50 \%$ of the patients for the consistencies tested. When the peak frequency, mean wave time, presence of residue in between swallowings, and acoustic signal suggestive of residues were correlated with decannulation (Table 5 ), no significant correlation was verified $(p>0.05)$ between the variables analyzed in the two consistencies and the decannulation.

Conclusion: the study suggests that it is feasible to use the Doppler sonar as a combined method in the clinical assessment of dysphagia for the decannulation of patients affected by traumatic brain injury.

Keywords: Brain Injuries; Tracheostomy; Deglutition Disorders; Doppler Effect 


\section{INTRODUCTION}

Traumatic brain injuries (TBI) have their severity classified with electroencephalography (EEG) as mild, moderate, or severe. It is currently, the most used parameter worldwide to assess the level of awareness. One of its main advantages is a set of physical examinations easy to use ${ }^{1,2}$.

The presence of a cannula, especially if the cuff is inflated, reduces the normal larynx movement, possibly causing atrophy for lack of use of the laryngeal musculature, an inability to generate subglottal air pressure, and the desensitization of the larynx and vocal folds ${ }^{3}$. As the cough reflex is reduced, changes may appear in the safety of the tracheostomized patient's swallowing biomechanics ${ }^{4}$. All these factors favor food and/or saliva to enter the lower respiratory tract - prevailing the silent aspiration, which leads to an impairment of the swallowing dynamics ${ }^{5,6}$.

The assessment of swallowing using an instrument in combination with the clinical examination contributes decisively to define the subsequent procedures. Mechanisms that make such practice feasible require that new methodologies be created, tested, and explored. The Doppler sonar is an innovative, noninvasive, radiation-free, easy to use, low-cost method ${ }^{7}$. Figures 1, 2, and 3 show the graphic representation of the swallowing sounds, picked up by the Doppler sonar, in the normal swallowing of food in pudding and liquid consistency, respectively.

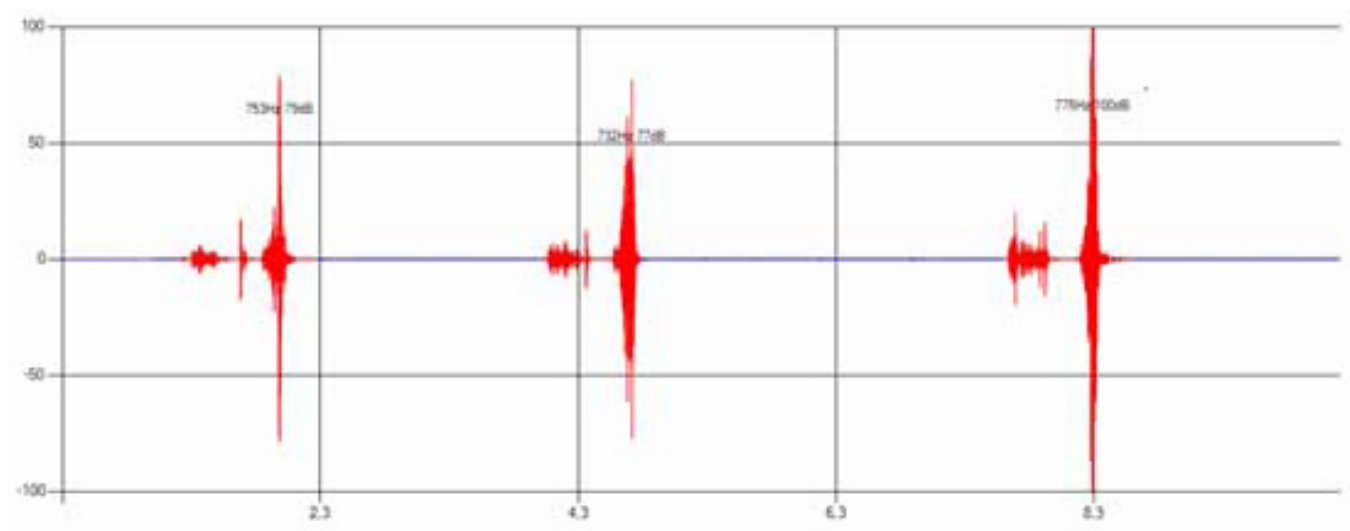

Number of swallowings: 3 Mean time: $1.0 \mathrm{~s}$ Mean peak frequency: $753 \mathrm{~Hz}$ Intensity: $85.4 \mathrm{~dB}$ Organized signal

Source: The author (2016)

Figure 1. Graphic representation of the variables of the swallowing sounds picked up with the Doppler sonar

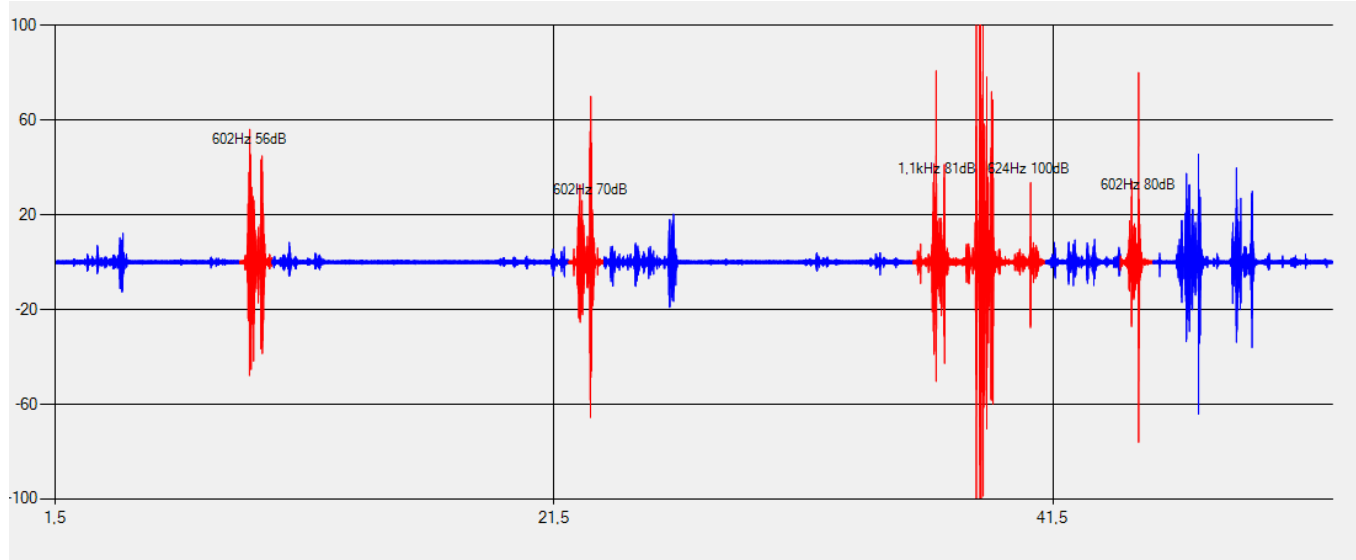

Source: The author (2016)

Figure 2. Graphic representation of the variables of the swallowing sounds picked up with the Doppler sonar, for pudding food consistency 


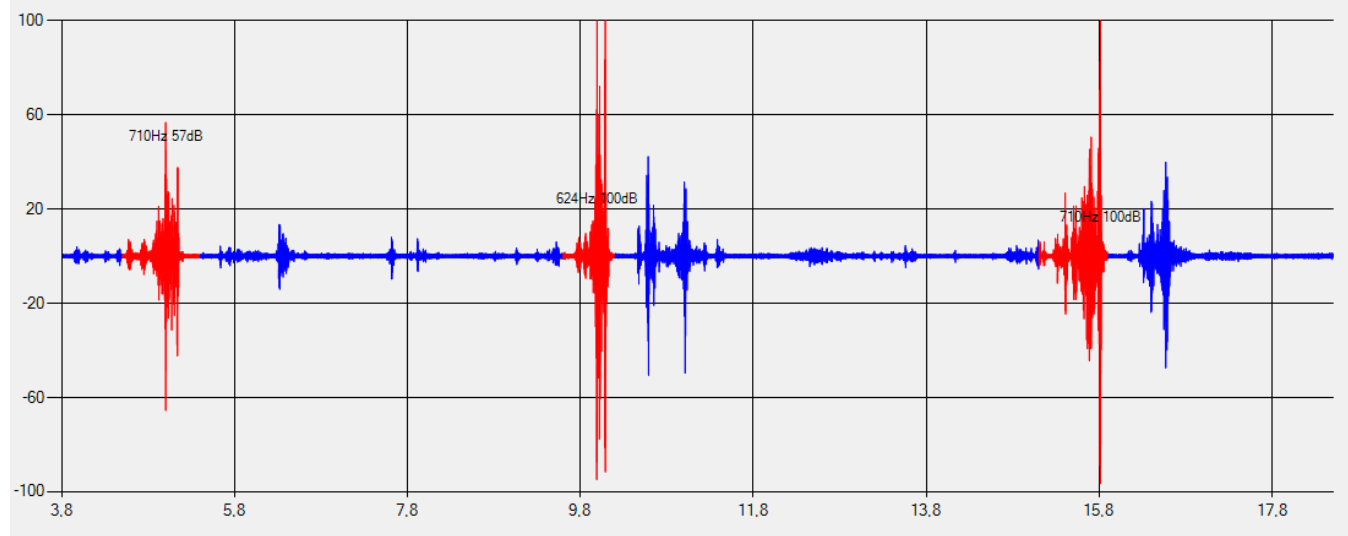

Source: The author (2016)

Figure 3. Graphic representation of the variables of the swallowing sounds picked up with the Doppler sonar, for liquid food consistency

This study aimed to demonstrate the feasibility of using acoustic analysis of swallowing sounds as a method combined with clinical assessment in tracheostomized patients affected by TBI.

\section{METHODS}

This is an observational, cross-sectional study, approved by the Human Research Ethics Committee of the Hospital Instituto Paranaense de Otorrinolaringologia, Brazil, under number 1.453.832. The data for the research was collected in the Department of Neurology of the Hospital do Trabalhador, with hospitalized patients in wards, semiintensive, or intensive care units. They were assessed when required and/or allowed by the responsible physician. The medical records were analyzed in the hospital archive.

The sample comprised 10 patients hospitalized in the Intensive Care Unit (ICU) between May and July 2016, affected by TBI, which was confirmed with a CT scan. They were tracheostomized and their feeding was exclusively enteral. Eight (80\%) patients were males and 2 (20\%), females, mean age 43.6 years, and standard deviation 21.48 years. Patients without an exam confirmation, or who did not agree to participate in the research were excluded. The degree of the TBI was assessed by the medical team and was registered in the medical record; all of them were severe.

The assessment consisted of applying the Speech Therapy Tracheal Decannulation Protocol (STDP) ${ }^{8}$, based on the analysis of the patients' identification data present in the medical records, considering the variables: age, sex, diagnosis, the anatomical region of the lesion, and degree of the TBI. Once the patient had met the requirements, the STDP was applied. In the assessment conducted by the speech-languagehearing therapist, six tracheal decannulation criteria were observed, as described below:

1. Level of awareness: The patients were observed in terms of the score in the Glasgow coma scale, in which a score lower than eight was considered insufficient for the protection of the respiratory tract and, consequently, for decannulation ${ }^{2,8-10}$.

2. Breathing: During the assessment, the type of cannula was observed (whether it was a metal or plastic one), the presence of cuff, and whether it was inflated or not. The patients that could not keep their cuff deflated were not assessed, leaving it to a more adequate moment. It was observed that those whose cuff remained deflated maintained a breathing pattern when the cannula was occluded, hence continuing the assessment process. The breathing pattern was considered maintained when the patient could keep the blood oxygen saturation rates (SpO2) above 90\% $\%^{3,9}$.

3. Orotracheal secretion: It was subjectively analyzed by the speech-language-hearing therapist, as there were no objective methods to measure and classify orotracheal secretion, regarding the volume, aspect, and color of the secretion present in the orotracheal region. The aspect of the secretion (whether thick or fluid) present in the orotracheal region or aspirated indicated the level of hydration or humidification. the clear color indicated an absence of infection, while the yellowish indicated infection ${ }^{9,10}$.

4. Phonation: Initially, it was observed whether the patient was verbally responsive. In the affirmative 
cases, they were asked to emit a sustained /e/ vowel to assess the presence of "wet" vocal quality. When they presented a "wet" voice (after swallowing), the patient was asked to spontaneously clear the laryngopharynx without risk of tracheal aspiration. Physiologically, the "wet voice" is a consequence of vocal production with prandial material in the larynx, changing the mass in the vocal folds and hence the person's voice quality. Vocal change after swallowing has been pointed out as a parameter to detect dysphagia in clinical assessment ${ }^{8,11}$.

5. Swallowing: For the functional clinical assessment of swallowing, the patient was either sitting or in bed with the back lifted to $90^{\circ}$ and the tracheostomy occluded. They were given three spoonfuls of pudding-consistency food, thickened in $100 \mathrm{ml}$ of water, with $3.6 \mathrm{~g}$ of ThickenUp Clear; starch mix, with the following nutritional value for 125g: $347 \mathrm{kcal}, 83 \mathrm{~g}$ of carbohydrates, and 1354 mg of sodium; and three spoonfuls (10 $\mathrm{ml}$ each) in liquid consistency.

6. Coughing: The presence of voluntary coughing was observed; it was considered effective only when the patient could expel the material from the respiratory tract while the food was being given or after it.

To pick up the sound, a portable ultrasonic detector was used, model DF-4001, manufactured by Martec, with a single-crystal, flat-disc transducer, furnishing interface to the Doppler sonar. The ultrasound frequency was $2.5 \mathrm{~Hz}$, with an output of $10 \mathrm{~mW} / \mathrm{cm}^{2}$ and a sound power of $1 \mathrm{~W}$. The continuous Doppler device was linked to a notebook computer HP Pavilion g4-1115br, Processor AMD Dual-Core, 500 GB hard disk memory, 3 GB RAM, high color support video card, CD-ROM drive, sound card, and speakers.

The operating system used was Windows ${ }^{\circledR}$ Seven $^{\circledR}$ and the software, DeglutiSom ${ }^{\circledR 12}$. DeglutiSom ${ }^{\circledR}$ is a swallowing sound analysis software, developed in a partnership between speech-language-hearing pathologists and engineers, to pick up and analyze the acoustics of the swallowing sounds to assess, monitor, and rehabilitate cases of oropharyngeal dysphagia.

The transducer beam was positioned to form an angle from $30^{\circ}$ to $60^{\circ}$. Nine variables were measured: Initial frequency of the sound wave (Fol), frequency of the first peak of the sound wave (FoP1), frequency of the second peak of the sound wave (FoP2), initial and final intensity of the sound wave (II) and (FI), swallowing time $(\mathrm{T})$, pause time between the sucking and swallowing sequences (TSu) and (Tsw), and time elapsed from beginning to end of the acoustic signal analyzed, measured with the audio signal, in seconds.

\section{RESULTS}

Answering the speech therapy protocol for tracheal decannulation, all the patients scored above 11 in Glasgow. The literature suggests a score above eight as ideal for the assessment ${ }^{2,8,10}$.

The metallic cannula was found in $60 \%$ of the patients, and the plastic one, in $40 \%$ of those assessed. All the patients had a respiratory pattern when the tracheostomy was closed with the presence of orotracheal secretion and effective coughing in $90 \%$. The orotracheal secretion had a small volume in $80 \%$, fluid viscosity, and clear color in $90 \%$ of the patients. Regarding responsiveness, $70 \%$ of the patients were verbally responsive, and $60 \%$ did not have a wet voice. At the end of the assessment, $70 \%$ proved to be apt to decannulate.

Tracheostomized individuals have changes in the swallowing process, as swallowing and breathing are closely related. The presence of a cannula can change the relationship between these functions, predisposing to dysphagia and tracheal aspiration ${ }^{6,13}$.

In this sense, a specialized assessment is necessary to the process of removing the cannula, which must begin when the causes that lead to the indication of tracheostomy have been solved. Tracheal decannulation is essential to the person's rehabilitation and improvement in the quality of life, observing clinical criteria to minimize risks of failure. Despite its importance, authors have not yet reached a consensus about the criteria that need to be assessed ${ }^{14,15}$. 
Table 1. Results of the acoustic analysis for the pudding food consistency

\begin{tabular}{lccccc}
\hline VARIABLE & N & MEAN & MINIMUM & MAXIMUM & $\begin{array}{c}\text { STANDARD } \\
\text { DEVIATION }\end{array}$ \\
\hline Peak frequency & 10 & $665.50 \mathrm{~Hz}$ & $589.00 \mathrm{~Hz}$ & $786.00 \mathrm{~Hz}$ & $56.70 \mathrm{~Hz}$ \\
Mean intensity & 10 & $63.60 \mathrm{~dB}$ & $41.00 \mathrm{~dB}$ & $80.00 \mathrm{~dB}$ & $10.76 \mathrm{~dB}$ \\
Mean wave time & 10 & $1.59 \mathrm{~s}$ & $1.04 \mathrm{~s}$ & $2.37 \mathrm{~s}$ & $0.44 \mathrm{~s}$ \\
Time in between swallowings & 10 & $5.45 \mathrm{~s}$ & $0.80 \mathrm{~s}$ & $14.00 \mathrm{~s}$ & $4.88 \mathrm{~s}$ \\
\hline
\end{tabular}

Source: The author (2016)

The acoustic analysis of the pudding food swallowing sounds was based on three swallowings. The data refer to standard deviation, frequency of the peak in Hertz $(\mathrm{Hz})$, mean intensity in decibels $(\mathrm{dB})$, mean wave time in seconds (s), and time in between swallowings (s).

Table 2. Results of the frequencies of the acoustic analysis for pudding food consistency

\begin{tabular}{lcc}
\hline VARIABLE & NO & YES \\
\hline Acoustic signal of laryngeal elevation & $5(50 \%)$ & $5(50 \%)$ \\
Presence of noise in between swallowings & $5(50 \%)$ & $5(50 \%)$ \\
Presence of acoustic signal suggestive of residue & $5(50 \%)$ & $5(50 \%)$ \\
Presence of acoustic signal suggestive of aspiration & $8(80 \%)$ & $2(20 \%)$ \\
Coughing & $10(100 \%)$ & $-(0 \%)$ \\
Apnea & $10(100 \%)$ & $-(0 \%)$ \\
\hline
\end{tabular}

Source: The author (2016)

It was observed that $50 \%$ of the individuals analyzed presented acoustic signals of laryngeal elevation, presence of noise between pudding food swallowings, and presence of acoustic signals suggestive of residue;
$80 \%$ did not present signals suggestive of aspiration and laryngotracheal penetration, and none of the patients had coughing or apnea.

Table 3. Results of the acoustic analysis for the liquid food consistency

\begin{tabular}{lccccc}
\hline VARIABLE & N & MEAN & MINIMUM & MAXIMUM & $\begin{array}{c}\text { STANDARD } \\
\text { DEVIATION }\end{array}$ \\
\hline Peak frequency & 10 & $719.60 \mathrm{~Hz}$ & $642.00 \mathrm{~Hz}$ & $851.00 \mathrm{~Hz}$ & $66.89 \mathrm{~Hz}$ \\
Mean intensity & 10 & $75.40 \mathrm{~dB}$ & $61.00 \mathrm{~dB}$ & $99.00 \mathrm{~dB}$ & $12 . \mathrm{dB}$ \\
Mean wave time & 10 & $1.37 \mathrm{~s}$ & $1.02 \mathrm{~s}$ & $1.89 \mathrm{~s}$ & $0.37 \mathrm{~s}$ \\
Time in between swallowings & 10 & $5.23 \mathrm{~s}$ & $0.80 \mathrm{~s}$ & $22.00 \mathrm{~s}$ & $6.58 \mathrm{~s}$ \\
\hline
\end{tabular}

Source: The author (2016)

In the acoustic analysis for liquids, based on three swallowings of $5 \mathrm{ml}$ each, the mean peak frequency was $719.60 \mathrm{~Hz}$, with a standard deviation of $66.89 \mathrm{~Hz}$; mean intensity of $75.40 \mathrm{~dB}$, with a standard deviation of $12.91 \mathrm{~dB}$; mean wave time $1.37 \mathrm{~s}$, with a standard deviation of $0.37 \mathrm{~s}$; time in between swallowings was of $5.23 \mathrm{~s}$, with a standard deviation of $6.58 \mathrm{~s}$. 
Table 4. Results of the frequencies of the acoustic analysis for liquid food consistency

\begin{tabular}{lcc}
\hline VARIABLE & NO & YES \\
\hline Acoustic signal of laryngeal elevation & $5(50 \%)$ & $5(50 \%)$ \\
Presence of noise in between swallowings & $5(50 \%)$ & $5(50 \%)$ \\
Presence of acoustic signal suggestive of residue & $5(50 \%)$ & $5(50 \%)$ \\
Presence of acoustic signal suggestive of aspiration & $8(80 \%)$ & $2(20 \%)$ \\
Coughing & $10(100 \%)$ & $-(0 \%)$ \\
Apnea & $10(100 \%)$ & $-(0 \%)$ \\
\hline
\end{tabular}

Source: The author (2016)

It was observed that $50 \%$ of the individuals analyzed presented acoustic signals of laryngeal elevation, presence of noise between liquid food swallowings, and presence of acoustic signals suggestive of residue;
$80 \%$ did not present signals suggestive of aspiration and laryngotracheal penetration, and none of the patients had coughing or apnea.

Table 5. Relationship between peak frequency, mean wave time, presence of residue in between swallowings, acoustic signal suggestive of residues and decannulation

\begin{tabular}{|c|c|c|c|c|c|c|}
\hline \multirow{3}{*}{ VARIABLE } & \multicolumn{3}{|c|}{ PUDDING CONSISTENCY } & \multicolumn{3}{|c|}{ LIQUID CONSISTENCY } \\
\hline & \multicolumn{2}{|c|}{ Decannulation } & \multirow{2}{*}{$\mathrm{p}$-value } & \multicolumn{2}{|c|}{ Decannulation } & \multirow{2}{*}{ p-value } \\
\hline & No & Yes & & No & Yes & \\
\hline \multicolumn{7}{|l|}{ Peak frequency } \\
\hline Less than $700 \mathrm{~Hz}$ & 2 & 6 & 0.5333 & 1 & 4 & 0.5000 \\
\hline $700 \mathrm{~Hz}$ or more & 1 & 1 & & 2 & 3 & \\
\hline \multicolumn{7}{|l|}{ Mean wave time } \\
\hline Up to $1.50 \mathrm{~s}$ & 3 & 3 & 0.1667 & 3 & 3 & 0.1667 \\
\hline More than $1.50 \mathrm{~s}$ & - & 4 & & - & 4 & \\
\hline \multicolumn{7}{|c|}{$\begin{array}{l}\text { Presence of residues in } \\
\text { between swallowings }\end{array}$} \\
\hline No & 1 & 4 & 0.5000 & 1 & 4 & 0.5000 \\
\hline Yes & 2 & 3 & & 2 & 3 & \\
\hline \multicolumn{7}{|c|}{$\begin{array}{l}\text { Acoustic signal suggestive } \\
\text { of residues }\end{array}$} \\
\hline No & 1 & 7 & 0.0667 & 1 & 7 & 0.0667 \\
\hline Yes & 2 & - & & 2 & - & \\
\hline
\end{tabular}

Source: The author (2016)

Fisher's test

The relationship of food consistencies, swallowing acoustic findings, and the possibility of the person proceeding to tracheal decannulation was assessed. It was then observed that six of the decannulated patients had safe swallowing for pudding food, while four were safe for liquid food, with frequencies equal or superior to $700 \mathrm{~Hz}$.

When the mean wave time was assessed (normal up to $1.5 \mathrm{~s}$, and changed above $1.5 \mathrm{~s}$ ), an equal number of decannulated people was found for both consistencies tested. Such matching can be cited when related to the presence of residues in between swallowings and acoustic signals suggestive of residues and decannulation in any of the food consistencies studied.

Using Fisher's test, no significant relationship was found $(p>0.05)$ between the variables analyzed in the two consistencies and decannulation (Table 5). Such a result indicated that the hypothesis of there being an association between the variables can be dismissed. However, in clinical terms, based on 
the graphic representation of the swallowing sound variables picked up with the Doppler sonar, the bedside assessment of the patient can be performed with more criteria, as with its use it is possible to verify sound variations that suggest changes in the swallowing process, thus complementing the speech-languagehearing assessment.

\section{DISCUSSION}

The literature has currently referred to the acoustic analysis of the swallowing sounds with ultrasound, the Doppler sonar, as a possible mechanism to assist the speech-language-hearing therapist to define a diagnosis when assessing oropharyngeal dysphagia, as well as in the removal of tracheostomy. Such a method is based on the swallowing sounds, whose audible cues can help establish a reliable classification to work as a screening system to identify signals suggestive of laryngeal aspiration and penetration ${ }^{16}$.

Although there are not many standards for tracheostomy weaning, the decision to decannulate is still based on subjective assessments concomitantly with standardized protocols. Therefore, signals such as saliva or food residue in the trachea, change in the secretion color or aspect, fever, worse oxygenation, and lower level of awareness must be warning signs for all professionals involved in the patient's treatment ${ }^{3,6,12}$.

ASHA recommends that the clinical assessment of swallowing be conducted with all food consistencies. However, due to the severe condition of the researched patients, it was decided to assess them only with pudding and liquid consistencies ${ }^{17}$.

Studies suggest that, if in swallowing higher frequency and intensity and a shorter time correspond to a more efficient performance in the swallowing process $^{12,18}$, it is to be concluded that in this study the performance of the swallowing motor action is changed for pudding food consistency (Figure 2), which is not the case for the liquid consistency (Figure 3), corroborating the literature in question. The multiple characteristics of the swallowing sounds depend directly on the food consistency, which causes difficulties when preparing and organizing the food bolus, its slow handling, ejection difficulties, and a decreased anteroposterior tongue movement. Hence, food consistency and volume interfere with the swallowing process ${ }^{7}$. The slowness of the swallowing process can be even caused by the presence of the tracheostomy. When physiologically analyzed, its use brings about an important pressure change in the swallowing process.
Also, the weight of the tracheostomy cannula can impair the laryngeal elevation, possibly changing the normal swallowing process and leading to dysphagia, which may even be transitory ${ }^{3}$. Studies also report that the longer the orotracheal intubation, evolving to tracheostomy, the more severe the dysphagia will $b^{10,19}$. In the pudding food consistency, the mean peak frequency was observed, representing the cricopharyngeal opening with less intensity. It was possibly due to the slow opening, demonstrating a decreased wave sound amplitude for this food consistency.

The mean wave time and intensity also presented values below what is considered normal, as stated in the literature (Table 1). As for the liquid food consistency, the mean peak frequency, mean intensity and mean wave time presented values within normality $(\text { Table } 3)^{18}$.

When the peak frequency, mean wave time, presence of residue in between swallowings, and acoustic signal suggestive of residues were correlated with decannulation (Table 5), no significant correlation was verified $(p>0.05)$ between the variables analyzed in the two consistencies and decannulation. The literature indicates that most of the procedures used in clinical examinations that assess the swallowing function are not sensitive and specific to define tracheal aspiration ${ }^{12}$. Thus, combining functional assessment and acoustic analysis of swallowing, the speechlanguage-hearing therapist can reach a more certain diagnosis of aspiration. The present study does not demonstrate significant values when analyzing the variables of the sound signal in relation to the presence of an acoustic signal of residues and the possibility of tracheal decannulation. It is believed that noise variations in the laryngeal region may hinder the distinction of the sound signal referring to food or saliva residues present in that region.

Cervical auscultation with Doppler sonar enabled the pharyngeal phase to be analyzed with a noninvasive, low cost, easily and quickly performed intervention, with no radiation exposure. The intervention caused the patients no discomfort, hence making it possible to obtain various swallowing samples, either sequential or not. This method allowed the assessment data to be retaken many times and in different moments. Thus, a relationship was established with its physiology, a factor that contributed to the speech-language-hearing assessment. It furnished numerical, objective, and documentable data, portraying reliable information. 
The acoustic analysis of swallowing with Doppler sonar has proved to be a promising technique in this field, as it combines speed, agility, and low cost, besides being painless, with no radiation exposure, and wholly noninvasive. Moreover, it avoids unnecessary displacements in the case of patients with some sort of restriction ${ }^{12}$.

Regarding the quality of the audio in the acoustic analysis, some factors need to be observed when it is being performed. For instance, excessive movements must be avoided during the examination, as well as the inadequate positioning to pick up the audio, ultrasonic detector poorly attached to the skin, or even technical problems in the equipment. Previous studies involving acoustic analysis of swallowing had already demonstrated these types of setbacks. They also pointed to the need to exclude signals inadequate to an appropriate analysis, due to the presence of strong disturbances, such as speech sounds, coughing, and excessive head movement ${ }^{20}$. Given the above, and the difficulties found during the data collection, it is suggested that the study be continued with a larger sample, whose results provide a greater basis and scientific evidence.

As it is a portable device, the bedside assessment was made easier. The acoustic analysis of swallowing proved to be an auxiliary tool in decision-making concerning the decannulation of tracheostomized patients, helping to proceed to the other instrumental examinations. It could be used to monitor the treatment of swallowing disorder, providing biofeedback to the patient. Hence, they had the opportunity to see and resee their collected tests, comparing them to the recordings made during the therapies ${ }^{12,16,18}$.

\section{CONCLUSION}

In practical terms, the Doppler sonar is feasible as an auxiliary method in the clinical assessment of dysphagia to decannulate tracheostomized patients affected by traumatic brain injury.

\section{REFERENCES}

1. Teasdale G, Jennet B. Assessment of coma and impaired consciousness, a practical scale. J Lancet. 1974;2(7872):81-4.

2. Zanata IL, Santos RS, Marques JM, Hirata GC, Santos DA. Speech-language pathology assessment for tracheal decannulation in patients suffering from traumatic brain injury. CoDAS. 2016;28(6):710-6.
3. Furmann N, Costa FM. Clinical criteria used by health professionals for release oral diet in hospitalized adult patients. Rev. CEFAC. 2015;17(4):1278-87.

4. Garuti G, Reverberi $C$, Briganti A, Massobrio M, Lombardi F, Lusuardi M. Swallowing disorders in tracheostomised patients: a multidisciplinary/ multiprofessional approach in decannulation protocols. Multidiscip respir med. 2014;9(1):36.

5. Furkim AM, Duarte $S$, Flores FT, Santos RS, Nunes MCA. Evaluacion clínica de las disfagias orofaringeas. In: Franklin S, Marchesan I, Parra D, Dioses A (orgs). Tratado de evolucion de motricidade orofacial. EOS. 2014. p. 233-46.

6. Santana L, Fernandes A, Brasileiro AG, Abreu AC. Criteria for clinical speech pathology evaluation of tracheostomized patient in hospital and home. Rev. CEFAC. 2014;16(2):524-36.

7. Soria FS, Silva RG, Furkim AM. Análise acústica da deglutição orofaríngea utilizando Sonar Doppler. Braz. j. otorhinolaryngol. 2016;82(1):39-46.

8. Zanata IL, Santos RS, Hirata GC. Tracheal decannulation protocol in patients affected by traumatic brain injury. Arch otolaryngol. 2014;18(2):108-14.

9. Andrade AG, Lima GM, Albuquerque JWA, Anijar NP, Teixeira RC. Decannulation process in patients afflicted with traumatic brain injury: study performed in a hospital trauma, in the metropolitan region of Belém, PA, Brazil. Rev Fac Ciênc Méd. 2017;19(4):196-200.

10. Medeiros GC, Sassi FC, Silva CL, Andrade CRF. Criteria for tracheostomy decannulation: literature review. CoDAS. [serial on the Internet] 2019 [cited 2019 Aug 15];31(6).[about 9 p.]. Available from: https://www.scielo.br/pdf/codas/v31n6/2317-1782codas-31-6-e20180228.pdf

11. Santos KW, Scheeren B, Maciel AC, Cassol M. Postswallow voice modification: compatibility with videofluoroscopy findings. CoDAS [serial on the internet] 2017 [cited 2019 Aug 15]; 29(6) [about 6 p.]. Available from: https://www.scielo.br/pdf/ codas/v29n6/2317-1782-codas-29-6-e20170004. pdf

12. Santos RS, Abdulmasshi EM, Zeigeilboim BS, Teive HAG. 1.043 analysis of swallowing sounds through sonar doppler in patients with Parkinsons disease. Parkinsonism relat disord. 2012;18(Suppl 2):1-250.

13. Bernardi SA. Cânulas traqueais e disfagia orofaríngea: uma revisão integrativa da literatura. 
Rev. Bras. de Cirurgia de Cabeça e Pescoço. 2016;44(4):187-93.

14. Costa CC. Decannulation: speech therapy and physiotherapy approach. Distúrb. Comunic. 2016;28(1):93-101.

15. Pasqua F, Nardi I, Provenzano A, Mari A. Weaning from tracheostomy in subjects undergoing pulmonary rehabilitation. Multidisciplinary Respiratory Medicine. 2019;10(1):1-7.

16. Madalozzo B, Aoki MC, Soria F, Santos RS, Furkim AM. Acoustic analysis of swallowing time through Doppler Sonar. Rev. CEFAC. 2017;19(3):350-9.

17. ASHA (American Speech Language Hearing Association). Special interest division 13. Atlanta, GA. Swallowing and swallowing disorders. 1998.

18. Abdulmassih SEM, Teive HGA, Santos RS. The evaluation in patients with spinocerebelar ataxia and oropharyngeal dysphagia: spinocerebelar ataxia and oropharyngeal dysphagia: a comparison of videofluoroscopic and sonar Doppler. Arch otolaryngol. 2013;17(3):66-73.

19. Ferrucci JL, Sassi FC, Medeiros GC, Andrade CRF. Comparison between the functional aspects of swallowing and clinical markers in ICU patients with Traumatic Brain Injury (TBI). CoDAS [serial from Internet].2019 [cited 2019 Sep 10];31(2) [about 11 p]. Available from: https://www.scielo.br/pdf/codas/ v31n2/2317-1782-codas-31-2-e20170278.pdf

20. Sejdic E, Steele CM, Chau T. Segmentation of Dual-Axis Swallowing Accelerometry Signals in healthy subjects with analysis of anthropometric effects on duration of swallowing activities. IEEE Transactions on Biomedical Engineering. 2009;56(4):1090-7. 